
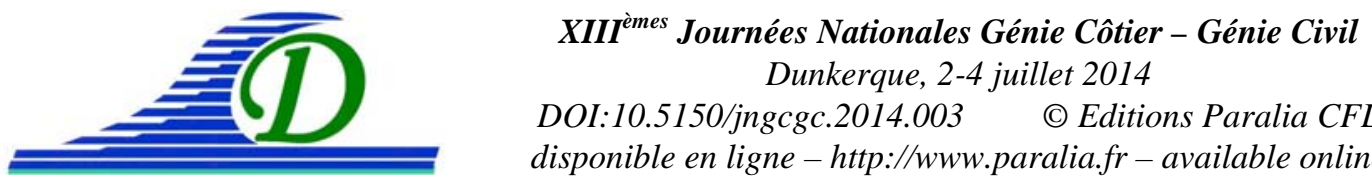

DOI:10.5150/jngcgc.2014.003 (C) Editions Paralia CFL

disponible en ligne - http://www.paralia.fr - available online

\title{
Ecoulements stationnaires ou oscillants à travers un milieu poreux : effets de la surface spécifique
}

\section{Gwendoline ARNAUD ${ }^{1,2}$, Damien SOUS ${ }^{1}$, Julien TOUBOUL ${ }^{1}$, Fabrice GOUAUD ${ }^{2}$, Vincent REY ${ }^{1}$}

\author{
1. Aix Marseille Université, CNRS/INSU, IRD, Mediterranean Institute of \\ Oceanography (MIO), UM 110, 13288 Marseille / Université de Toulon, \\ CNRS/INSU, IRD, Mediterranean Institute of Oceanography (MIO), UM 110, \\ F-83957 La Garde, France. \\ arnaud@univ-tln.fr ; sous@univ-tln.fr ; touboul@univ-tln.fr ; rey@univ-tln.fr, \\ 2. ACRI-In, 260, route du Pin Montard Sophia-Antipolis F-06410 Biot, France. \\ Fabrice.Gouaud@acri-in.fr
}

\section{Résumé :}

L'objet de ce travail était d'étudier l'effet de la surface spécifique sur la dissipation d'énergie à travers un milieu poreux "modèle", constitué d'un réseau dense de cylindres verticaux émergeants. Les expériences ont été menées dans un canal hydraulique à surface libre de longueur utile 10 mètres. Trois maquettes ont été réalisées à l'aide de cylindre de diamètres variables. Chacune de ces maquettes, d'une longueur maximale de $4.80 \mathrm{~m}$, est constituée d'un réseau régulier de cylindres de diamètre constant. Ce dispositif nous permet d'étudier, à porosité constante égale à 0.7 , l'effet des variations de surface spécifique sur l'écoulement. Une première série de mesure a été réalisée pour des courants stationnaires, dans des conditions de vitesse et de hauteurs d'eau variables, une deuxième série sur la propagation de houles régulières. Les résultats en présence d'un courant stationnaire ont montré une influence très significative de la surface spécifique sur la perte de charge à travers le poreux. Cette influence se retrouve sur l'atténuation de l'onde et sur le phénomène d’interférence observé, d’autant plus faible que la structure est longue par rapport à la longueur d'onde. La longueur d'onde dans le milieu poreux est bien décrite par la porosité et le choix adéquat d’une masse ajoutée.

\section{Mots-clés :}

Ecoulement, Houle, Réflexion, Dissipation, Milieu poreux, Surface Spécifique.

\section{Introduction}

Les ouvrages du type structure poreuse sont largement étudiés et utilisés en génie côtier pour leur capacité à dissiper significativement l'énergie incidente de la houle. Les études d'efficacité de ces ouvrages, généralement menées à partir de modèles physiques en bassin et de modèles numériques, font apparaître la porosité comme un paramètre majeur. La surface spécifique, définie comme étant la surface de contact fluide-solide par unité de volume (GUYON et al., 1991) est un autre paramètre caractéristique des 


\section{Thème 1 - Hydrodynamique côtière}

milieux poreux. Si son rôle peut être implicitement pris en compte via des coefficients d'atténuation, ce paramètre en particulier dans le phénomène de dissipation n'est pas explicité en génie côtier. Il peut être pourtant significatif, puisque pour des écoulements permanents lents, la perméabilité d'un milieu, proportionnelle au cube de la porosité, est également inversement proportionnelle au carré de la surface spécifique (GUYON et al., 1991). Pour les études de propagation de houle au dessus de milieux poreux, les effets d'atténuation sont le plus souvent liés à des effets inertiels et non-linéaires (GU \& WANG, 1991 et références citées). Sur la base des travaux de SOLLITT ET CROSS (1972), la prise en compte de ces effets conduit à une expression de la relation de dispersion complexe, qui traduit un régime de propagation avec dissipation. YU \& CHWANG (1994) ont étudié le cas de poreux superposés, pour des conditions faiblement à fortement dissipatives, en tenant compte des modes "évanescents" dus à la présence de discontinuités d'indice du milieu dans la direction de propagation de l'onde. Les modèles précédents se basent sur une approche linéaire des effets de dissipation. Pour des écoulements à plus haut nombre de Reynolds, MOLIN (2011) a proposé une approche quadratique pour des milieux poreux de faible épaisseur.

Le travail présenté a pour but de mieux comprendre les rôles respectifs de la porosité et de la surface spécifique dans les processus de dissipation. Une première série de mesure a été réalisée pour des courants stationnaires, dans des conditions de vitesse et de hauteurs d'eau variables, une deuxième série sur la propagation de houles régulières. Les résultats sont présentés successivement dans la section 3 après une présentation du dispositif expérimental dans la section 2. Une discussion des résultats et les perspectives de travaux sont présentées dans la section 4.

\section{Dispositif expérimental}

\subsection{Canal hydrodynamique et milieu poreux}

Le canal hydrodynamique de SeaTech (ex-ISITV), Université de Toulon, possède une longueur utile de 10 mètres et une largeur efficace de $0.3 \mathrm{~m}$ (voir figure 1). La hauteur d'eau maximale est de $0.50 \mathrm{~m}$. Il peut être utilisé comme canal hydraulique ou canal à houle.

Le milieu poreux "modèle" est constitué d'un réseau dense de cylindres verticaux émergeants, disposés régulièrement selon deux axes perpendiculaires faisant un angle de $45^{\circ}$ avec l'axe longitudinal du canal. Trois diamètres $D$, respectivement $0.020,0.032$ et $0.050 \mathrm{~m}$, ont été considérés. La porosité $\gamma$ à travers les cylindres est constante et égale à 0.7 . La surface spécifique $s$ prend les valeurs 52,33 et $22 \mathrm{~m}^{-1}$ pour les valeurs croissantes des diamètres. La longueur maximale du milieu poreux était de $4.80 \mathrm{~m}$. 


\section{XIII ${ }^{\text {èmes }}$ Journées Nationales Génie Côtier - Génie Civil \\ Dunkerque, 2-4 juillet 2014}

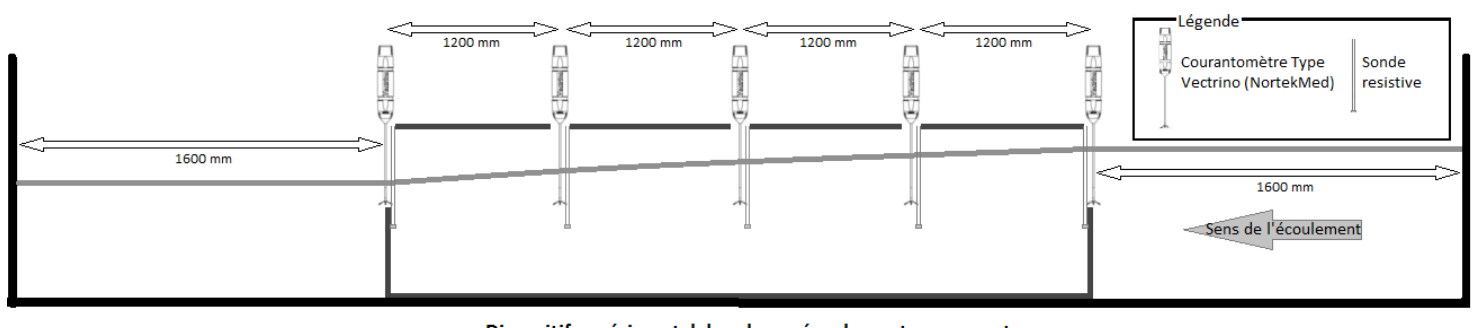

Dispositif expérimental dans le cas écoulement permanent

Figure 1. Canal hydrodynamique et dispositif expérimental pour le cas écoulement permanent.

\subsection{Conditions d'expériences et instrumentation}

Le niveau de la surface libre était mesuré à l'aide de 7 sondes résistives synchrones, avec une fréquence d'échantillonnage de $32 \mathrm{~Hz}$, les vitesses par 5 courantomètres acoustiques doppler de type Vectrino (fourni par NortekMED), avec une fréquence d'échantillonnage de $200 \mathrm{~Hz}$. Le volume d'échantillonnage des courantomètres est d'environ $10 \mathrm{~mm}^{3}$.

Dans le cas de l'écoulement permanent, la longueur de la structure poreuse était $L=4.80 \mathrm{~m}$. Le canal hydraulique est équipé d'un tube de Pitot qui permet de déterminer le débit associé à la pompe du canal. Ce débit est commandé par l'ouverture de la vanne de la pompe. Les courantomètres étaient positionnés respectivement à l'entrée du poreux et puis tous les $1.20 \mathrm{~m}$ jusqu'à la sortie du poreux. L'acquisition de ces 5 courantomètres a été synchronisée sur le premier Vectrino de la série placé en amont de la structure. Les sondes résistives, pour la mesure du niveau d'eau, étaient disposées au niveau des différents courantomètres. Nous avons étudié des débits compris entre $3 \times 10^{-3}$ et $16 \times 10^{-3} \mathrm{~m}^{3} / \mathrm{s}$, pour différents niveaux d'eau (vitesses comprises entre 0.04 et $0.20 \mathrm{~m} / \mathrm{s})$.

Les études en présence de houle ont été réalisées pour deux longueurs $\mathrm{L}$ du poreux, respectivement $1.20 \mathrm{~m}$ et $2.40 \mathrm{~m}$, par profondeur d'eau $h=0.23 \mathrm{~m}$. Des études de réflexion, transmission et atténuation de la houle ont été menées dans la gamme de périodes 0.55 - $2 \mathrm{~s}$. Trois sondes, distantes respectivement de $0.3 \mathrm{~m}$ et $0.5 \mathrm{~m}$, à $0.90 \mathrm{~m}$ du milieu poreux, ont été disposées en amont et en aval de la structure. Les calculs ont été réalisés par la méthode à trois sondes (MANSARD \& FUNKE, 1980). Les courantomètres ont également été installés de part et d'autre et dans le poreux.

\section{Résultats expérimentaux}

\subsection{Perte de charge pour un écoulement permanent}

Pour des écoulements lents, le débit $Q$ à travers une surface $S$ en fonction de la perte de charge $\Delta P$ est de la forme : 
$Q=U . S=-\frac{K}{\mu} \frac{\Delta P}{L}$

où $\mu$ est la viscosité du fluide, $U$ la vitesse moyenne de l'écoulement et $K$ la perméabilité du milieu poreux. Pour des écoulements plus rapides, la vitesse n'est plus proportionnelle au gradient de pression, qui peut alors s'écrire :

$\frac{\Delta P}{L}=\alpha U+\beta U^{2}$

Nous avons représenté sur la figure 2 la perte de charge en fonction du débit. Nous constatons que le rapport $\alpha /(\beta U)$ est inférieur à 0.2 dans le cas des cylindres de diamètre 0.032 et $0.05 \mathrm{~m}$ et il peut atteindre la valeur de 0.5 pour $D=0.020$. La perte de charge varie donc quasiment comme le carré de la vitesse. Les effets inertiels sont prépondérants notamment pour les plus gros diamètres.

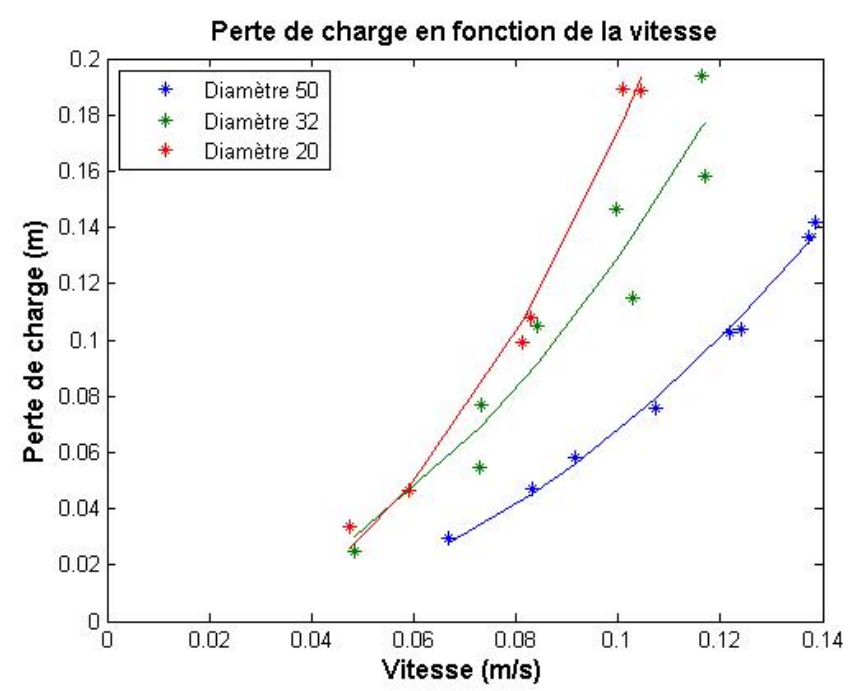

Figure 2. Perte de charge en fonction de la taille des cylindres, écoulement permanent.

\subsection{Impact sur la propagation d'une houle : réflexion et atténuation}

Les résultats concernant les coefficients de réflexion et de transmission sont présentés sur les figures 3 à 8, pour les trois poreux considérés, et les deux longueurs L étudiées. Ils sont comparés à des calculs basés sur une approche linéaire.

\subsubsection{Le modèle linéaire}

On suppose que la houle est de faible amplitude, et que le mouvement induit dans le fluide dérive d'un potentiel des vitesses. On considère que la dissipation de l'onde se fait sur une échelle d'espace suffisamment grande pour permettre de l'exprimer sous la forme d'un terme d'atténuation exponentiel dans la direction de propagation. En écrivant pour chacun des trois domaines $j, j=1,2.3$, respectivement amont, milieux 


\section{XIII ${ }^{\text {èmes }}$ Journées Nationales Génie Côtier - Génie Civil \\ Dunkerque, 2-4 juillet 2014}

poreux et aval, l'expression générale du potentiel des vitesses sous la forme (propagation selon les x positifs ou négatifs) :

$\Phi_{j}(x, z, t)=\phi_{j}(x, z) e^{i(\omega t)}=A_{j} e^{ \pm\left(i k_{j} x\right)} \cosh \left(k_{j}(z+h)\right) e^{i(\omega t)}$

avec $k_{j}=k_{j, p}+\mathrm{i} k_{j, d}$ le vecteur d'onde de l'onde, complexe dans le poreux avec un terme dissipatif, les coefficients $A_{j}$ sont les inconnues.

L'origine des abscisses correspond au début du milieu poreux. Le terme dissipatif est supposé nul de part et d'autre du poreux. Il est choisi de la forme $k_{j, d}=k_{j, p} / n$ dans le poreux, ce qui correspond à un taux d'atténuation constant par unité de longueur d'onde, d'autant plus grand que $n$ est petit. En amont du poreux, l'onde est une combinaison des ondes incidentes et réfléchie, en aval, l’onde est supposée progressive (pas de réflexion de la plage). Dans le poreux, l'onde est la superposition d'une onde progressive et d'une onde réfléchies, dont la propagation s'accompagne d'une dissipation. Les continuités des vitesses et des pressions aux interfaces $x=0$ et $x=L$ imposent :

$\phi_{i}=\gamma \phi_{j}$, et $: \frac{\partial \phi_{i}}{\partial x}=S_{r} \frac{\partial \phi_{j}}{\partial x}$

avec $i$ indice correspondant au milieu amont $(x=0)$ ou aval $(x=L), j$ indice du milieu poreux. $S_{r}$ est la réactance, elle est définie par :

$S_{r}=1+C_{m} \frac{\gamma-1}{\gamma}$

avec $C_{m}$, coefficient de masse ajoutée, $C_{m}=0$ pour les milieux amont et aval. La relation de dispersion est de la forme générale, en ne conservant que les effets inertiels (YU \& CHWANG, 1994) :

$\omega^{2} S_{r}=g k_{i} \tanh \left(k_{i} h\right)$

où $g$ est l'accélération de la pesanteur et $\omega$ la pulsation de la houle.

La longueur d'onde de l'onde dans le milieu poreux dépend de la valeur de $C_{m}$, qui sera calculée à partir des résultats expérimentaux. Les équations sont résolues à partir d'une méthode intégrale (YU \& CHWANG, 1994).

\subsubsection{Résultats}

Les résultats expérimentaux obtenus à partir des sondes résistives synchrones sont reportés sur les figures 3 à 8 . Les coefficients de réflexion $R$, de transmission $T$, ainsi que le coefficient de réflexion $R_{p}$ de la plage sont présentés. On peut remarquer qu'à longueur donnée du milieu poreux, la dissipation est d'autant plus importante que la taille des cylindres est faibles, et que cette dissipation est la plus grande aux hautes fréquences. Concernant la réflexion, celle-ci présente un aspect oscillant en fonction de la fréquence, caractéristique d'un phénomène d'interférence, dû à deux sauts d'indice successifs, au début $(x=0)$ et à la fin du poreux $(x=L)$. Les maxima de réflexion apparaissent lorsque les ondes incidente et réfléchie depuis la fin du milieu poreux 


\section{Thème 1 - Hydrodynamique côtière}

$(x=L)$ sont en phase au début du poreux $(x=0)$, ce qui explique, pour une plage de fréquences données, un nombre supérieur d'oscillations pour le poreux le plus long. Le processus d'interférence est d'autant mieux observé que la taille des cylindres est importante ou que la longueur du poreux est faible. Ceci s'explique par l'atténuation de l'onde au cours de sa propagation à travers le poreux, qui après un aller-retour (distance de parcours $2 L$ ) est atténuée et donc interfère d'autant moins avec l'onde incidente. L'oscillation observée tend à disparaitre pour de grandes valeurs de $L$, le coefficient de réflexion étant alors égale à 0.2 environ quelle que soit la fréquence.

L'oscillation du coefficient de réflexion dépend donc de la longueur d'onde de l'onde. Celle-ci peut être calculée théoriquement à partir de la formule (6). On observe qu'en faisant varier le coefficient $C_{m}$, la longueur d'onde varie. De même la diminution du coefficient de transmission est lié au terme d'atténuation $k_{2, d}=k_{2, p} / n$, où l'indice 2 fait référence au milieu poreux. Nous avons donc fait varier les paramètres $C_{m}$ et $n$. Les résultats théoriques présentés sur les figures 3 à 8 sont obtenus avec $C_{m}=0.3$ et $n=13,20$, 30 respectivement pour $D=0.020,0.032,0.050 \mathrm{~m}$.

On observe un bon accord entre théorie et expérience pour le coefficient de réflexion, et son caractère oscillant en fonction de la fréquence. Nous avons choisi de caler la courbe d'atténuation sur la partie haute de fréquence, dont la décroissance mesurée correspond bien à celle calculée. La loi de décroissance par unité de longueur d'onde proposée est donc valable dans cette gamme de fréquence. Pour les fréquences inférieures à $1 \mathrm{~Hz}$, le comportement de la courbe expérimentale pour la transmission est tout autre, laissant apparaitre une atténuation indépendante de la fréquence. Concernant le phénomène d'interférence, un très bon accord entre expériences et théorie est observé pour un même choix du $C_{m}$, qui semble bien reproduire la physique associée à la propagation de l'onde pour toute la gamme de fréquence étudiée.

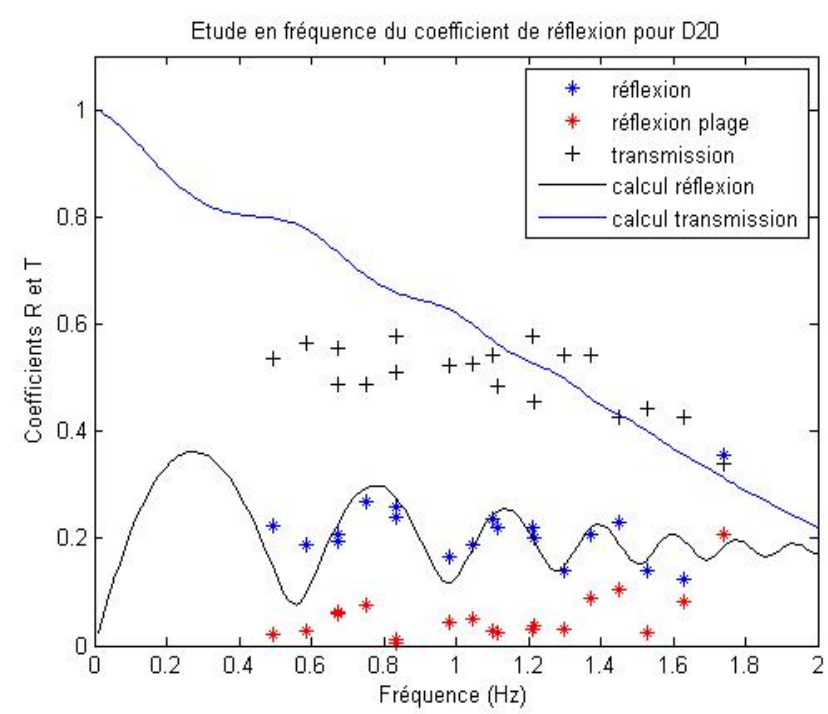

Figure 3. Coefficients de réflexion et de transmission, $L=1.20 \mathrm{~m}, D=0.020 \mathrm{~m}$. 


\section{XIII ${ }^{\text {èmes }}$ Journées Nationales Génie Côtier - Génie Civil \\ Dunkerque, 2-4 juillet 2014}

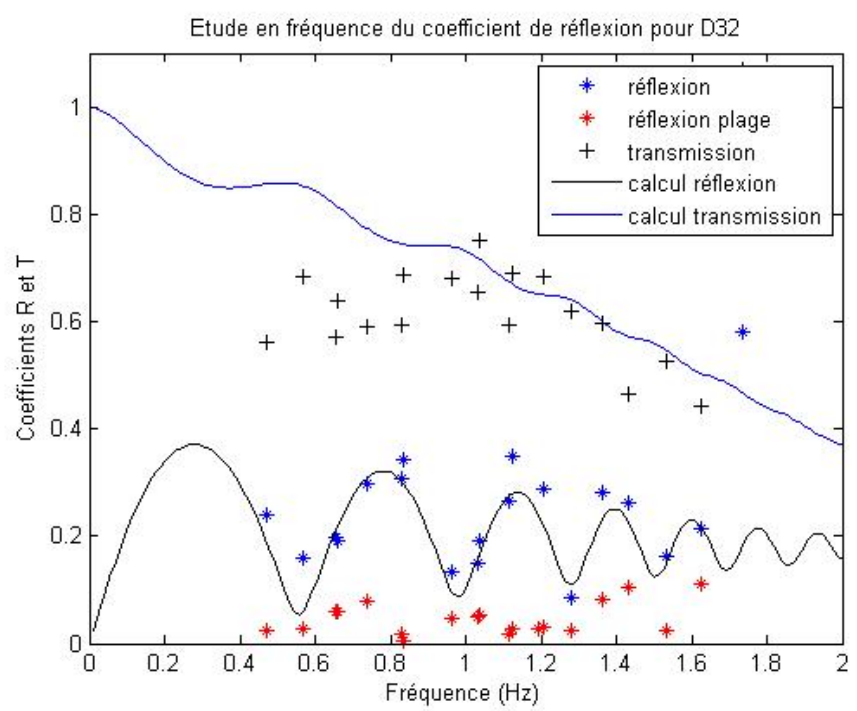

Figure 4. Coefficients de réflexion et de transmission, $L=1.20 \mathrm{~m}, D=0.032 \mathrm{~m}$.

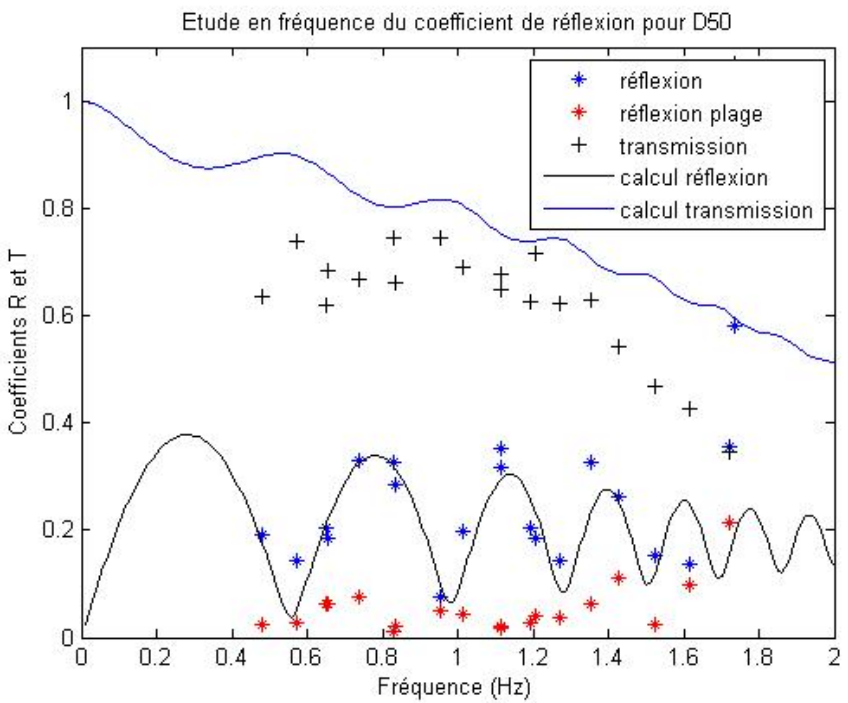

Figure 5. Coefficients de réflexion et de transmission, $L=1.20 \mathrm{~m}, D=0.050 \mathrm{~m}$. 
Thème 1 - Hydrodynamique côtière

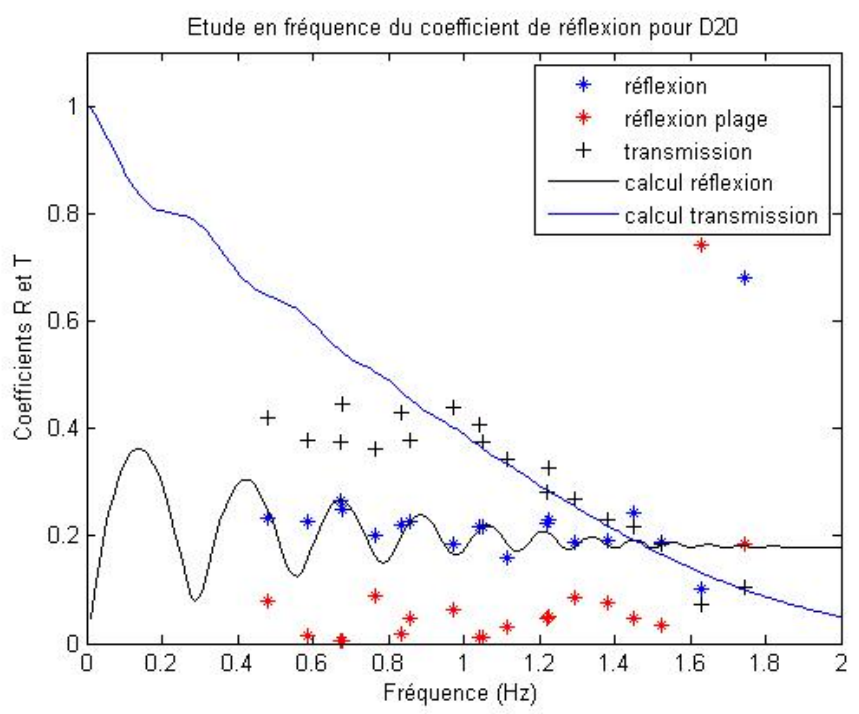

Figure 6. Coefficients de réflexion et de transmission, $L=2.40 \mathrm{~m}, D=0.020 \mathrm{~m}$.

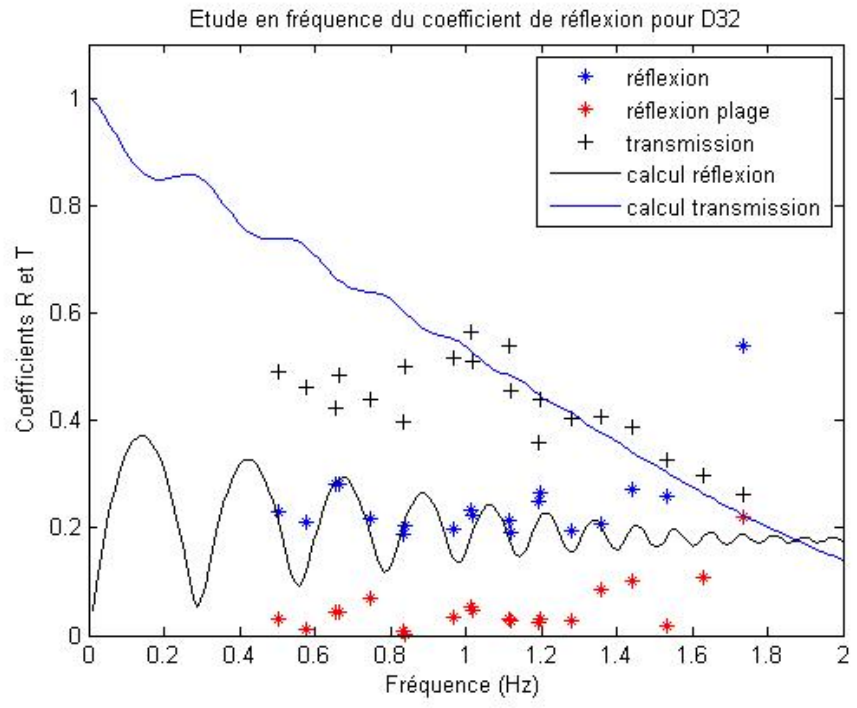

Figure 7. Coefficients de réflexion et de transmission, $L=2.40 \mathrm{~m}, D=0.032 \mathrm{~m}$. 


\section{XIII ${ }^{\text {èmes }}$ Journées Nationales Génie Côtier - Génie Civil \\ Dunkerque, 2-4 juillet 2014}

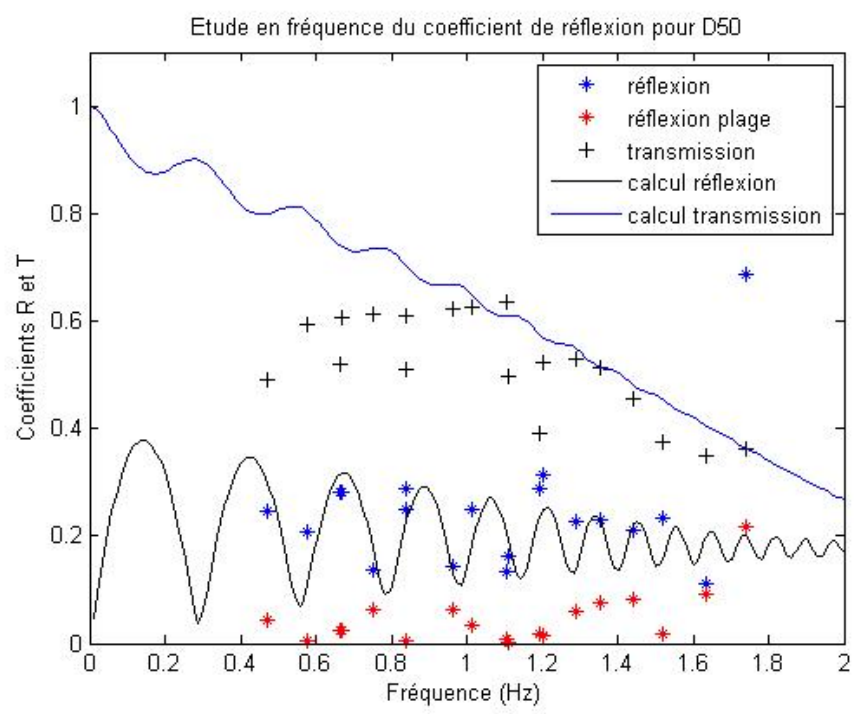

Figure 8. Coefficients de réflexion et de transmission, $L=2.40 \mathrm{~m}, D=0.050 \mathrm{~m}$.

\section{Conclusions et perspectives}

Le travail présenté montre l'influence significative de la surface spécifique sur la dissipation à travers le milieu poreux, constitué d'un ensemble dense de cylindres verticaux. Les expériences menées pour des écoulements permanents ont montré que les effets inertiels deviennent rapidement prépondérants, d'après une relation quasi-linéaire entre les gradients de pression et les carrés des vitesses. L'effet de la surface spécifique est également significatif pour les écoulements induits par la houle à travers le poreux. Les premiers résultats présentés ici montrent les rôles respectifs de la masse ajoutée associée à la porosité d'une part, qui pilotent le phénomène d'interférence, et de la dissipation associée à la surface spécifique d'autre part, qui contrôle l'atténuation de la houle.

Concernant les expériences avec la houle, outre les mesures de surface libre, des mesures de vitesses ont également été acquises lors de ces expériences. Par ailleurs le bon accord entre modèle et expérience doit permettre de calculer les champs de vitesses dans le fluide. La courbe de transmission de l'onde mesurée (et par conséquent de son atténuation) a montré deux zones, une première aux basses fréquences, peu sensible à la fréquence, une seconde à plus haute fréquence, complètement corrélée à cette dernière. Les calculs des vitesses locales, et des paramètres classiques (Reynolds, KeuleganCarpenter) sont prévus pour mieux comprendre et quantifier les processus à l'origine de cette atténuation. Un des buts sera d'y associer le paramètre de surface spécifique, en vue d'étude de facteurs d'échelle associés aux études en modèles réduits.

\section{Références}

GU Z., WANG H. (1991). Gravity waves over porous bottoms. Coast. Eng. Vol. 15, pp 497-524. http://dx.doi.org/10.1016/0378-3839(91)90025-C 
Thème 1 - Hydrodynamique côtière

GUYON E., HULIN J.P., PETIT L. (1991). Ecoulements dans les milieux poreux. In: Hydrodynamique Physique, Collection Savoirs Actuels, Interédition/édition Du CNRS. pp 375-384.

MANSARD E.P.D., FUNKE E.R. (1980). The Measurement of Incident and Reflected Spectra Using a least Squares Method. In: Proc. 15th Coastal Engineering Conference, Vol. 1. pp 154-172.

MOLIN B. (2011). Hydrodynamic modeling of perforated structures. Appl. Ocean Res., Vol. 33, pp 1-11. http://dx.doi.org/10.1016/j.apor.2010.11.003

SOLLITT C., CROSS R., 1972. Wave transmission through permeable breakwaters. Coast. Eng. Proc. Vol. 1, pp 1827-1846.

YU X., CHWANG A.T. (1994). Wave motion through porous structures. J. Eng. Mech. Vol. 120, pp 989-1008. http://dx.doi.org/10.1061/(ASCE)0733-9399(1994)120:5(989) 